\title{
Inverting the blowtorch: studying the 'slow' molecular kinetics of proteins with nonequilibrium response spectroscopy
}

\author{
MARK M. Millonas \\ Department of Pharmacological and Physiological Sciences, The University of Chicago, \\ MC6094, 5841 South Maryland Avenue, Chicago, IL 60637, U.S.A.
}

\begin{abstract}
Nonequilibrium response spectroscopy (NRS) is a novel method of analyzing the molecular kinetics of voltage-sensitive proteins that involves measurement of the nonequilibrium response of the molecules driven in a thermodynamically irreversible way by fluctuating fields with large amplitudes and frequencies. NRS is sensitive to features of the conformational kinetics that can not be resolved by means of more traditional relaxation transient type experiments, and can be understood conceptually as an inversion of Landauer's blowtorch idea.
\end{abstract}

(c) 1998 Academic Press Limited

Key words: molecular kinetics, ion channels, nonequilibrium response.

\section{Introduction}

One of Rolf Landauer's contributions to statistical physics that has had the most influence on my way of thinking is the so-called 'blowtorch' principle [1,2]. This, in many way, is a contribution made over an entire career (see the reference in [1]), and so is a fitting point to start a paper in honor of this career. Over the years this principle has helped to tie many types of nonequilibrium phenomena together for me, and I am still impressed by the way that this implicitly [2], or in very simplified terms, captures the essence of the many important and interesting features of nonequilibrium systems by saying what nonequilibrium systems are not like. Perhaps because it has been expressed in such simple terms, and in the form of a criticism of existing approaches, the legacy of the blowtorch principle is often neglected in the literature. To mention only a single example, the most important physical principles 'discovered' by the currently fashionable ratchets craze [3] can reasonably be called footnotes to the blowtorch.

Landauer's 'blowtorch' principle states that the behavior of a nonequilibrium system will generally depend on the specific details of its kinetics, even on pathways that traverse infrequently occupied kinetic states far from any stable state. In contrast microscopically reversible systems can be well characterized by criteria that depend only on the local neighborhood of the equilibrium state. The original purpose of the blowtorch principle was to point out the fundamental problems with attempts to describe the nonequilibrium system via simple generalizations of the local treatments used to describe equilibrium systems. However, it truly expresses the source of the underlying complexity that has been discovered (an sometimes rediscovered) recently in areas such as noise-induced transitions, the theory of large fluctuations, and fluctuation-induced transport. Like many very general principles it tells us not so much what the answers are, but what is often more important, where to look. Here I will show how the blowtorch idea can be turned around: the nonequilibrium response of a system that is driven in a thermodynamically irreversible way can be used as a tool for uncovering subtle details of its underlying kinetics.

This idea has been used to study the kinetics of voltage-sensitive ion-channel proteins in the laboratory. An 


$$
\begin{aligned}
& \mathrm{I}_{4} \underset{\beta_{2}}{\stackrel{\alpha_{2}}{\rightleftharpoons}} \mathrm{I}_{5} \underset{\beta_{2}}{\stackrel{\alpha_{2}}{\rightleftharpoons}} \mathrm{I}_{1} \underset{\beta_{4}}{\stackrel{\alpha_{4}}{\rightleftharpoons}} \mathrm{I}_{2} \\
& \alpha_{5} \mid\left[\beta_{5} \quad \alpha_{6}|| \beta_{6} \alpha_{7}|| \beta_{7}\right. \\
& \mathrm{C}_{1} \underset{\beta_{1}}{\stackrel{u^{2} \alpha_{1}}{\rightleftharpoons}} \mathrm{C}_{2} \underset{u \beta_{1}}{\stackrel{u \alpha_{1}}{\rightleftharpoons}} \mathrm{C}_{3} \underset{u^{2} \beta_{1}}{\stackrel{\alpha_{1}}{\rightleftharpoons}} \mathrm{C}_{4} \underset{\beta_{2}}{\stackrel{\alpha_{2}}{\rightleftharpoons}} \mathrm{C}_{5} \underset{\beta_{3}}{\stackrel{\alpha_{3}}{\rightleftharpoons}} \mathrm{O}_{1} \underset{\beta_{4}}{\stackrel{\alpha_{4}}{\rightleftharpoons}} \mathrm{O}_{2}
\end{aligned}
$$

Fig. 1. Millonas-Hanck model of the human cardiac sodium channel gating kinetics. The voltage dependence of the rates is described in the text, and $u$ is a dimensionless parameter. Specific parameters are given in the footnote.

analysis of the structure and function of the biomolecules is important in understanding and controlling biological processes. However, the problem is even more difficult experimentally than the typical experimental physicist or chemist might realize. This is because many proteins exhibit their specific functional properties only in situ. Physicists can freeze protein molecules, zap them with lasers, and use many sophisticated techniques for measuring protein motions. While the study of protein dynamics in controlled settings may provide an interested physics laboratory for the study of a range of exotic phenomena [4], in most cases the biological applicability of information obtained under such conditions is limited. In order to explore biological structure-function a different course is usually taken involving what are sometimes severe experimental limitations. However, modern molecular biology has provided a host of powerful techniques based on recombinant DNA technology [5]. As the sophistication of these techniques grows biophysicists have started to seek an understanding and control of protein structure-function that extends beyond mere heuristic descriptions [6] to the underlying physical mechanisms themselves, and to dream of a physiologically meaningful way to make unambiguous experimental measurements of these properties.

To study severe experimental limitations. However, modern molecular biology has provided a host of powerful techniques basedon recombinant DNA technology [5]. As the sophistication of these techniques grows biophysicists have started to seek an understanding and control of protein structure-function that extends beyond mere heuristic descriptions [6] to the underlying physical mechanisms themselves, and to dream of a physiologically meaningful way to make unambiguous experimental measurements of these properties.

The study of voltage-gated ion channels, large transmembrane proteins in excitable cells that open a pore in response to electric fields, is a good example of this trend in molecular biophysics. Experimentalists would like to understand the location of the voltage sensors, the electrical properties, and the conformational substates of these proteins. The ion-channel-gating mechanism is believed to involve complex rearrangements in the tertiary structure of the protein. These conformational changes are dominated by thermal activation and occur on time scales of from microseconds to minutes. These are called 'slow' kinetics here to distinguish them from the 'fast' molecular motions that occur on the level of the individual atoms in the protein.

Biophysicists, like chemist, prefer to construct Markov models [7] such as the one pictured in Fig. 1. These models are used for studying and collating the 'slow' kinetic properties of ion channels. The states of these kinetic models are usually grouped (as in Fig. 1) into closed (C), open (O), and inactivated (I) conformations, where the transition rates $\alpha_{i}(V)$ and $\beta_{i}(V)$ between these states are voltage dependent. Transitions between the states are thermally activated events and typically are assumed to take the exponential form $\alpha_{i}(V)=$ $\alpha_{i}(0) e^{q_{i} \delta_{i} V / k T}$, and $\beta_{i}(V)=\beta_{i}(0) e^{-q_{i}\left(1-\delta_{i}\right) V / k T}$ that is in accord with Eyring rate theory, where $V$ is the voltage applied across the cell membrane, $\alpha_{i}(0)$ and $\beta_{i}(0)$ are the activation rates at zero voltage, $q_{i}$ are the gating charges, and $0<\delta_{i}<1$ are dimensionless parameters representing fractional electrical distances. These parameters determine a transition matrix $\tilde{\mathbf{W}}$ for a discrete master equation $\dot{\rho}(t)=\tilde{\mathbf{W}}[V] \rho$ that describes the evolution of the probability distribution vector $\rho(t)$ over the $n$ conformational states of the model. If such models are valid, the states and transition rates give information about the gross molecular conformations and electrical properties of the ion-channel protein. However, they should not necessarily be interpreted as representations of the actual molecular motions. For that the representation must be more or less unique, 
but as we will see, using conventional methods, it is not possible to resolve enough detail of the underlying kinetics to make such a unique interpretation possible.

The voltage-clamp technique, in which the voltage across a cell membrane is controlled by a feedback circuit [8] that balances (and therefore measures) the net current has been the best biophysical tool for the study of voltage-sensitive channels since the beginning of modern biophysics with the work of Hodgkin and Huxley [6]. Since its initial development [9], a basic set of voltage-clamp protocols based on potential stepping have dominated electrophysiological studies of the properties of ion channels. This is the situation in which the voltage across a membrane is stepped from a holding potential to a test potential, or at a few points in time, and the current transient recorded. Information about the electrophysiological properties of a channel is then obtained from an analysis of these relaxation transients since the relaxation of the probability distribution can be calculated formally from the master equation, $\rho(t)=\exp (t \tilde{\mathbf{W}}[V] \rho(0)$, where $\rho(0)$ is the initial distribution when the voltage is stepped to $V$ at $t=0$. This is the relaxation transient method. This situation is completely analogous to many experimental situations in condensed-matter physics. While the basis for much of electrophysiology, the relaxation transient method also sets some limitations. Very different kinetic models can lead to very similar relaxation transients [10], so these different kinetic representations are degenerate with respect to the conventional data. Therefore, what is needed is a new type of experimental approach that breaks this degeneracy.

\section{Critique of the relaxation transient method}

A generic analysis of such experiments which will first be present reveals the source of their limitations. By mapping relaxation transient experiments onto the appropriate space the feature of the experiments can be formally analyzed. What can then immediately be seen from this analysis is that these experiments are intrinsically ambiguous.

We wish to determine the elementary voltage-dependent rates of transition between the $n$ gross conformational states where in this case there are no means to independently determine these quantities. Analogous limitations arise in many different contexts, for example the limitations that arise in the analysis of the decay of a correlation function in any complex system. A measurement of transition rates requires ensemble measurements, and the state $\rho$ of an ensemble of channels is a point $(n-1)$-dimensional space (normalization requires $\left.\sum_{i} \rho_{i}=1\right)$, which we will call the kinetic manifold of the channel. Experimental measurements determine only certain projections of the distribution vector- $(n-1)$-to-1 mappings from the kinetic manifold to the observable current that are fixed by the physics of the molecule. The macroscopic ionic current (the quantity measured below) is equivalent to a measurement of the mean probability of the conducting (open) state or states $I=\sum_{i} g_{O_{i}} \rho_{O_{i}}$. Single-channel measurements can distinguish between states with different conductances $g_{O_{i}}$. Gating currents (displacement currents that result from movements of the gating charges) are measurements of the rate of change of the total gating charge $\dot{C}(t) \propto \boldsymbol{Q} \cdot \dot{\boldsymbol{\rho}}(t)$, where $Q_{i}=\sum_{j=1}^{i} q_{j}$.

The maximum amount of information can be extracted from a given data type by measuring the quantity on all possible ensemble states $\rho$, a situation that is also impossible since we cannot prepare an arbitrary ensemble state. The easiest distributions to prepare lie on the one-dimensional equilibrium submanifold (parametrized by $V$ ) of equilibrium states, $\rho_{e q}(V)$ (where $\tilde{\mathbf{W}}[V] \rho_{e q}(V)=\mathbf{0}$ ), by allowing the channels to relax at a fixed potential $V$. Further distributions can be prepared from this set of states by stepping $($ at $t=0)$ to the new voltage $V^{\prime}$. The channel ensemble executes a brief excursion away from the equilibrium submanifold before returning to it at the new point $\rho_{e q}\left(V^{\prime}\right)$. While this excursion is taking place the channel explores onedimensional trajectory, $\rho_{1}\left(V, V^{\prime}, t\right)=\exp \left(t \tilde{\mathbf{W}}\left[V^{\prime}\right]\right) \rho_{e q}(V)$. Since the points reached by stepped potential experiments are parametrized by not more than three parameters $\left(V_{0}, V^{\prime}\right.$, and $\left.t\right)$, when $n-1 \gg 3$, the set of all possible distributions reachable by stepped potential experiments (the one-step submanifold) is one of very large codimension with respect to the whole kinetic manifold. In other words, one-step experiments hardly 
explore the kinetic phase-space at all—the source of a great deal of ambiguity. Models based on data from these experiments are correspondingly ambiguous.

Standard relaxation transient experiments thus involve two independent limitations: one due to observational (projection) limitations and the other due to limitations of protocol. While a great deal of work has gone into removing the limitations of projection by developing new types of measurement, little work has been done on resolving the limitations of the relaxation transient method itself. Protocol ambiguities arise from limitations in our ability to experimentally explore all points in the kinetic phase-space. They might be removed by enlarging the region of that space explored by driving the channels off the one-step manifold. The logical extension of this idea is to change the voltage very rapidly at a large number of points in time, a method we call nonequilibrium response spectroscopy (NRS). In order to drive the channel ensemble off the one-step manifold the voltage must fluctuate more rapidly than the channel relaxes to equilibrium.

\section{An example of NRS}

The kinetic model shown in Fig. 1 was fit to data from whole-cell ionic current recordings from human cardiac sodium channels (hH1a) heterologously expressed in HEK293 cells. Macroscopic currents of the model in response to a voltage step are given by $I(t)=g(V)\left(V-V_{r}\right) \boldsymbol{e}_{O} \cdot \boldsymbol{\rho}(t)$, where $V_{r}$ is the reversal potential, $\boldsymbol{e}_{0}$ is the projection vector used to extract the probability of the open (conducting) states from $\boldsymbol{\rho}(t)$, and $g(V)$ is the instantaneous conductance that takes into account voltage dependencies of the conductance that are independent of gating. These include GHK rectification and voltage-dependent block of the channel by extracellular divalent cations like $\mathrm{Ca}^{+2}$. The reversal potential and $g(V)$ can be determined independently by experiment [11] using standard techniques. Our model is similar to one proposed for the sodium channels of the squid giant axon [12], except for additional open and inactivated states $\mathrm{O}_{2}$ and $\mathrm{I}_{2}$. The model in [12] was based on combined information from single-channel, macroscopic-ionic and gating-current recordings, and reproduced all of these types of data very well. It thus represents the state of the art with respect to modeling sodium channels. While some differences are to be expected there are major similarities because of the close homology, between the two isoforms. Model parameters ${ }^{\dagger}$ were determined by minimizing of the chi-squared error between the set of 10 stepped potential relaxation transients and the model predictions for these transients (Fig. 2A, B) [11]. The results were in good agreement with the finding of many other researchers that used data from the stepped potential series: in particular the transitions to the inactivated state and the last activation step before the channel opens are fairly voltage independent, while the most voltage-dependent steps are the first activation steps.

The homogeneous form of the kinetic equation makes calculating the evolution of the probability distribution simple when the voltage is held constant. This simplifying property can be preserved at the expense of allowing the voltage itself to fluctuate in a random but Markovian way. An example of this type of voltage noise is telegraph noise [13]: a stochastic process $V_{t}$ that switches at random times between two states, $V_{t} \in\left\{V_{+}, V_{-}\right\}$, at the mean rate $\omega / 2$. The time-dependent transition rates in the model are then given adiabatically by $\alpha_{i}(t)=\alpha_{i}[V(t)], \beta_{i}(t)=\beta_{i}[V(t)]$, an approximation that remains valid so long as the intraconformational fluctuation frequencies $(\simeq 80 \mathrm{kHz})$ [14] are much larger than the bandwidth $\omega$ of the voltage fluctuations ( $\leq 10 \mathrm{kHz}$ in the experiments described below) [11]. However, when the bandwidth of the voltage fluctuations is equivalent or greater than some or all of the interconformational transitions between states the response of the channel can become very sensitive to subtle features of the underlying kinetics. This again is an example of the blowtorch effect, though in this case the specific detail are somewhat removed from those considered

$\dagger$ The optimal paramer fit of the model to the stepped potential series (where in each case $i$ runs from 1 to 7 ) were: (in $\mathrm{kHz}) \alpha_{i}(0)=6.56,10.25,1.10,0.17,0.0092,2.37,13.96, \beta_{i}(0)=0.010,0.012,1.12,0.0085, *, 9.42007$; (in units of e) $q_{i}=3.65,3.82,0.041,0.11,{ }^{*}, 0.18,{ }^{*}$; (dimensionless) $\delta_{i}=0.03,0.60,0.56,0.24,{ }^{*}, 0.73,{ }^{*}$, where $q_{5} \delta_{5}=0.12$, and $q_{7} \delta_{7}=0.07, u=1.2$. $\mathrm{A}^{*}$ indicates that the parameters are constrained by conditions of microscopic reversibility. 

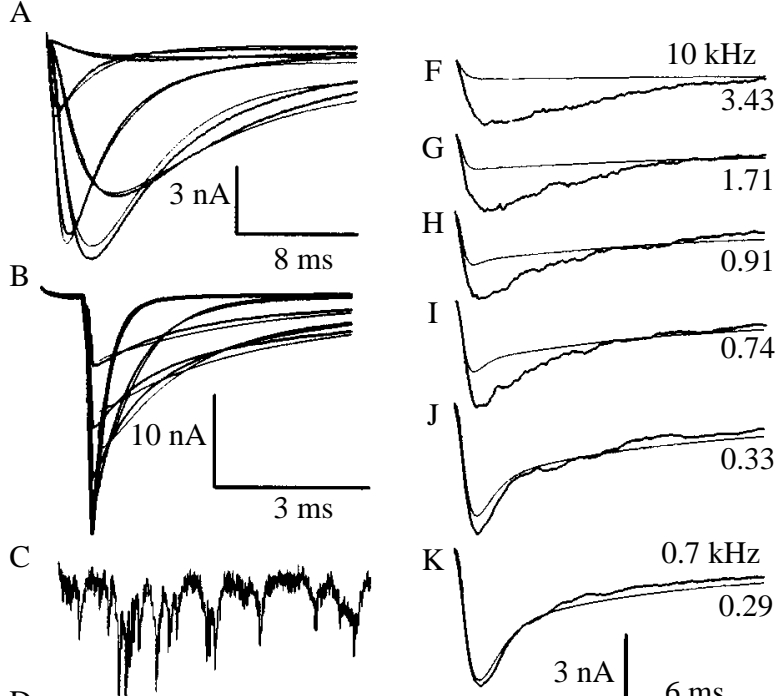

$\mathrm{D}$
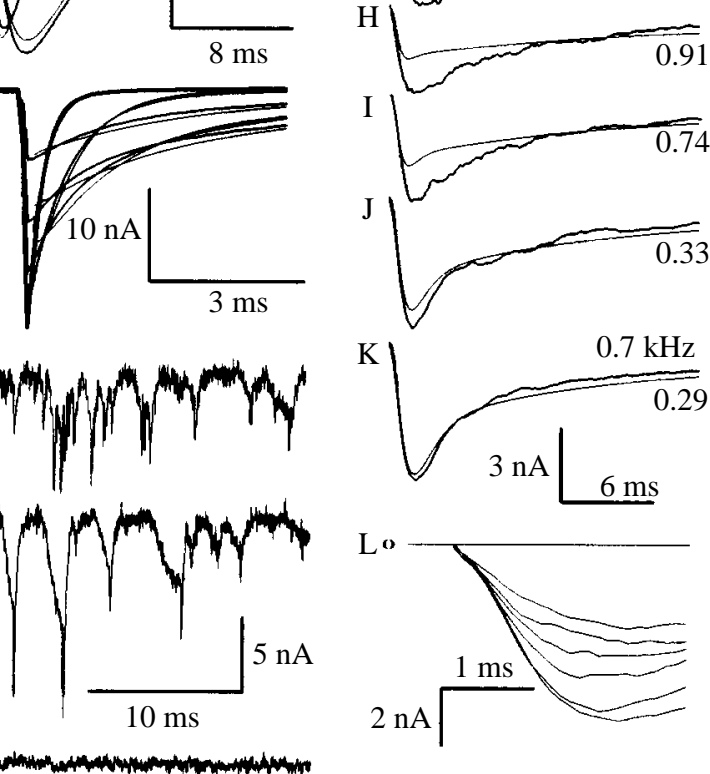

K

$0.7 \mathrm{kHz}$
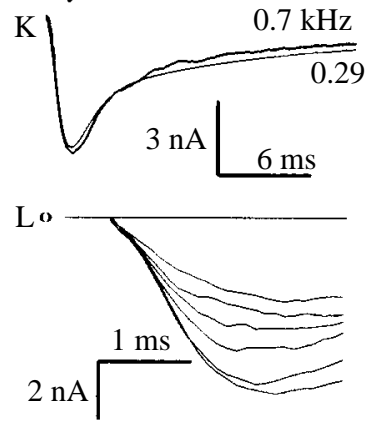

Fig. 2. Experiment showing NRS data is more sensitive than standard relaxation transient data. Model transients were calculated from the analytic expressions in the text using subroutines from Matlab. The reversal potential was $39.1 \mathrm{mv}$. All data from the same cell except for D, and carried out at $282 \mathrm{~K}$. A, B, Relaxation transients (heavy lines) and model predictions (thin lines) for steps from a holding potential of $-150 \mathrm{mV}$ to $\mathrm{A}-76,-58,-46,-22,14 \mathrm{mV}$ (activation) and $\mathrm{B}$ to $30 \mathrm{mV}$ for $1 \mathrm{~ms}$, and then to $-120,-90,-66,-42$, $-12 \mathrm{mV}$ (deactivation). The model was based on an optimal fit to the data shown in A and B. C, E, Raw current traces in response to voltage fluctuations with $V_{+}=-30 \mathrm{mV}$ and $V_{-}=-120 \mathrm{mV}$, and with bandwidths of C, E $10 \mathrm{kHz}$ and D $2 \mathrm{kHz}$. D control data from untransfected cell with no sodium channels. F-K Model predictions (thin lines) and NRS relaxation transients (heavy lines) formed by averaging 500 raw data traces such as $\mathrm{C}$ and D. Fluctuation band widths (in kHz): F, 10; G, 5; H, 3; I, 2; J, 1; K, 0.7. L. Superposition of first few $\mathrm{ms}$ of $\mathrm{F}-\mathrm{K}$ showing the decrease of the rise rate with increasing frequency.

by Landauer in his simple examples. As I have already mentioned, fluctuation-induced transport, a related effect, is a footnote to the blowtorch idea.

An $n$-state channel described by a kinetic scheme, such as pictured in Fig. 1, and driven by the telegraphnoise voltage fluctuations can be described by the $2 n$-dimensional conditional master equation $\dot{\wp}=\tilde{\mathbf{W}} \wp$, where

$$
\tilde{\mathbf{W}}=\left(\begin{array}{cc}
\tilde{\mathbf{W}}\left[V_{+}\right]-(\omega / 2) \mathbb{I} & (\omega / 2) \mathbb{I} \\
(\omega / 2) \mathbb{I} & \tilde{\mathbf{W}}\left[V_{-}\right]-(\omega / 2) \mathbb{I}
\end{array}\right)
$$

and where $\wp=\left(\rho_{+}, \rho_{-}\right)$is a $2 n$-dimensional conditional probability distribution vector, where $\boldsymbol{\rho}_{ \pm}$is the conditional probability vector given that the voltage is in the $V_{ \pm}$states. It may help to think of the system as an extended Markov model with states specified by both channel and voltage indices. The formal solution for the evolution of the conditional probability vector is $\wp(t)=\exp (t \tilde{\mathbf{W}}) \wp(0)$, where $\wp(0)$ is determined by the initial state of both the channel and the voltage. In all the experiments done here the initial state of the voltage was set to $V_{+}$, so $\wp(0)=(\rho(0), \mathbf{0})$. The simplest way to use this method to analyze whole-cell recordings is to compute averages over many different realizations of the individual fluctuating current traces in response 
to pulses of telegraph noise of a given duration. The mean transient whole-cell current is $\langle I\rangle=\left\langle I_{+}\right\rangle+\left\langle I_{-}\right\rangle$, where $\left\langle I_{ \pm}\right\rangle=g\left(V_{ \pm}\right)\left(V-V_{ \pm}\right) \boldsymbol{E}_{O}^{ \pm} \cdot \wp(t)$, and where $\boldsymbol{E}_{O}^{+}=\left(\boldsymbol{e}_{O}, \mathbf{0}\right)$ and $\boldsymbol{E}_{O}^{-}=\left(\boldsymbol{0}, \boldsymbol{e}_{O}\right)$. Information is also contained in the fluctuations of the individual current traces but is not as helpful from the standpoint of accurate kinetic analysis because, in contrast to the average current transients, only the one-dimensional stationary case corresponding to the outdated and quantitatively incorrect Hodgkin-Huxley model is amenable to a simple mathematical treatment [13].

Figure $2 \mathrm{~F}-\mathrm{M}$ shows mean transient responses to telegraph noise with bandwidths that vary from $700 \mathrm{~Hz}$ to $10 \mathrm{kHz}$ together with the corresponding predictions of our model. The experiment is designed so that the input bandwidth is high enough that its effect on the response is negligible. We are able to achieve such high-frequency voltage control by making use of a specially designed low-resistance pipette as discussed in more detail in [11]. The resistance of the pipettes in solution can be as low as $100 \mathrm{k} \Omega$. Similar techniques have been used in our laboratory for a number of years [15], and under certain conditions, when series resistance compensation is used, RC times of as little as $5 \mu$ s (corresponding to a corner frequency of $30 \mathrm{kHz}$ ) can be achieved. Here such high speeds were not required. The data shown were obtained with an input bandwidth just in excess of $10 \mathrm{kHz}$, and series resistance compensation was not used since all that was necessary to focus on the most interesting effects lie in the range between 2 and $6 \mathrm{kHz}$, which was well below our corner frequency.

Our model was based on stepped potential data and for low frequencies, as expected, continues to prove a good predictor of the mean current in line with the analysis presented above. Since we used activation and tail-current recordings that spanned the range of physiological potentials, the model represents nearly the best that can be done with a stepped potential series. Model parameters were consistent with the finding of other researchers.

For higher frequencies a mismatch begins to occur at frequencies of about $1-2 \mathrm{kHz}$, an indication of the discrepancy between the model and nature. This represents information invisible to relaxation transient analysis (Fig. 2A, B). After the analysis presented above it should not be surprising that this mismatch starts to occur in the region of most rapid change in the peak amplitude of the response, which nearly doubles when the frequency is lowered from 3 to $1 \mathrm{kHz}$. Over much higher and lower frequencies the peak inward currents change very little. The response saturates above $6-10 \mathrm{kHz}$, and effect that occurs when the correlation time of the fluctuating voltage is much less that the relaxation time of the channel. Note that this frequency is well below the input-corner frequency, and is just an expression of the highest frequencies of the channel kinetics. This case can be treated by replacing the inhomogeneous kinetic equation $\rho(t)=\tilde{\mathbf{W}}[V(t)] \rho$ by the homogeneous one, $\dot{\boldsymbol{\rho}}(t)=\langle\tilde{\mathbf{W}}\rangle_{V} \boldsymbol{\rho}$, where $\langle\tilde{\mathbf{W}}\rangle_{V}=\int \mathrm{d} V \rho_{0}(V) \tilde{\mathbf{W}}[V]$, where $\rho_{0}(V)$ is the stationary probability distribution of the voltage fluctuations. The response will be independent of all the features of the noise except the stationary probability density and therefore tractably analyzed for any kind of noise that has a sufficiently short correlation time. The model does not show the same saturation frequency as the data, another sign of the model's shortcomings that can not be seen by standard experimental techniques.

Attempts were made to optimize the model parameters by including NRS data from Fig. 2 F-M in the optimization set with the stepped potential data. We found that no model of the form shown in Fig. 1 can simultaneously reproduce all the data in Fig. 2. Thus the information obtained from NRS indicates that a rather different kind of kinetic scheme may be required to the one shown in Fig. 1. The ability of NRS to falsify models that can not be falsified with current techniques should provide a powerful new tool for increasing our understanding of the kinetics of voltage-sensitive proteins since the need to falsify oversimplified, nonunique models has been, and continues to be, behind the continual search for new and different ways to measure channel gating over the last half century.

$\tilde{\mathbf{W}}$ is the evolution operator of a microscopically reversible system, where as $\tilde{\mathbf{W}}$ is not. This difference in symmetry is at the root of the sensitivity of the method. When there are 'loops' in the kinetic diagram (as is the case here) the latter will evolve to a stationary distribution that lacks detailed balance. As is beginning to be understood, microscopically irreversible systems are capable of exhibiting many types of unusual behavior 


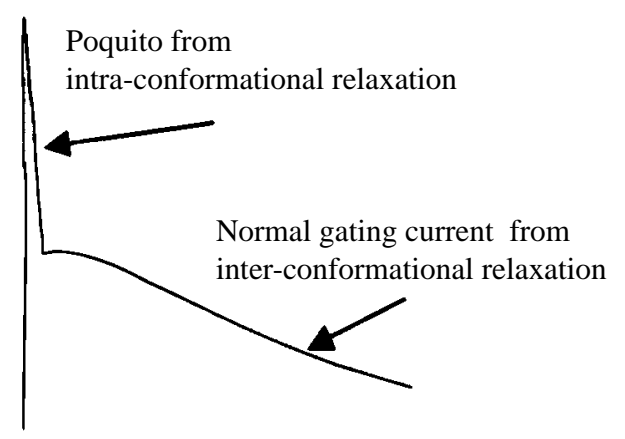

Fig. 3. Drawing of typical 'poquito' gating current resulting from a voltage step that sets the time scale of the intraconformational relaxation of the early activation states of the Shaker K.

that exhibit very sensitive, even singular $[13,16]$, dependence on specific details of the kinetics. The data in Fig. 2 E-M are just one example of this sensitivity. With very general arguments, as mentioned above, Rolf Landauer has emphasized similar points for a number of years [2, 1]. As far as I am aware this is the first time the idea has been used as an experimental tool for a more sensitive determination of the underlying kinetics of a system.

Since the master equation appears in many contexts in physics, chemistry and biology, analogous methods could be introduced to support model selection and kinetic analysis in these other contexts. In addition, the basic idea need not be restricted to the master equation, as will be shown in the next section. I believe these ideas should be of interest not only to experimental researchers studying protein dynamics, but also in the areas of statistical and nonlinear physics because they show theoretical ideas from these fields with a direct practical application to experimental biophysics. The experimental technique also provides an arena in which to explore nonequilibrium fluctuation-induced phenomena, and is therefore of interest on purely physical grounds.

\section{Ultra-high frequency NRS}

As shown above nonequilibrium response spectroscopy can be used for a more sensitive determination of the gating-kinetics ion channels than the relaxation-transient method. We might then be tempted to ask not just for a better way to probe the kinetics, but discover is there is a best, or optimal way. As discussed in the remainder of this paper, ultrahigh-frequency NRS is able to probe the microscopic details of the kinetics, and can be used to construct genuine physicochemical kinetic models for channel gating. Rather than serving as mere heuristic descriptions of the data from experiments, such models would fulfill the ultimate goal of molecular biophysics: a detailed microscopic understanding of the functionally important motions of biomolecules. For example, with such detailed microscopic models specific physicochemical descriptions of drug action could be formulated, and drug function could be both anticipated and engineered.

As will be discussed in more detail below, if high enough frequencies can be reached, shaped voltage pulses can be used to reveal the microscopic kinetics in a very precise way [17]. The required frequency is the same as the frequency of microconformational fluctuations. Recently Stefani and Bezanilla [18] at UCLA measured this time scale in gating-current measurements from the Shaker K channel. They found a short $2 \mu$ s wide spike of additional gating current (see Fig. 3) at the beginning of a voltage pulse. These spikes, nicknamed 'poquitos' or small spikes, are caused by the first microconformational relaxations of the channel. Thus the frequencies required to probe the microscopic kinetics are in the range of $80 \mathrm{kHz}$-not too far from the $30 \mathrm{kHz}$ than can already be achieved under some conditions in our laboratory. Without going into technical detail here it will be simply stated that it is most likely possible to reach such frequencies in the near future 
using home-made high-speed voltage-clamp circuitry together with some technological developments that have been made by fabricating low-resistance pipettes [11].

Ultrahigh frequency NRS is based on dynamical reconstruction, an idea that arises directly out of the theory of large fluctuation in statistical physics [19]. Dynamical reconstruction is also related to the conceptually distinct field of optimal control engineering. To understand the details of how dynamical reconstruction works, it is first necessary to discuss the different levels of modeling of channel gating, their meaning, and their interrelation.

The discrete Markov modeling methods, currently used to model the gating of ion channels, are the ones most appropriate to the sensitivity of normal electrophysiological experiments. However, using ultrahighfrequency NRS it would be possible to probe details of the kinetics on a fine enough scale that discrete Markov models will no longer be adequate approximations of the response. While it will be possible to determine the parameters for the discrete level of modeling more accurately than ever before, it will also be possible to learn much more, and the level of detail of the modeling process will have to be modified accordingly.

On a fine enough level the protein can be modeled as a mechanical system coupled to a viscous bath. The parts of the ion channel that determine gating involve concerted motions of large portions of the protein molecule, in contrast to the motion of just a few amino acids. We know this because such a large effective charge (about 12 elementary charges) moves through the field across the membrane when the channel opens. Under such circumstances the proper description of the motion of the voltage sensor is that of an over-damped Langevin equation

$$
\Gamma \dot{\boldsymbol{X}}=-\nabla H(\boldsymbol{X})+Q \boldsymbol{E}(t)+\boldsymbol{f}(t)
$$

where $\Gamma$ is a coefficient of friction, $\boldsymbol{X}$ is a generalized conformational coordinate vector, and $H(\boldsymbol{X})$ is a conformational energy function that depends on $\boldsymbol{X}$. There are two forces that act on the voltage sensor. The first is an external force $Q \boldsymbol{E}(t)$, where $\boldsymbol{E}(t)$ is the electric field (controlled by the voltage applied across the membrane) and $Q$ is the actual charge of the moving elements in the protein. Note that this formulation is easily generalized to dipole couplings to the electric field, even though for simplicity I do not explicitly consider this scenario here. The second force that acts on the protein is a random force $f(t)$ due to the thermal fluctuations of the heat bath, where the components of $\boldsymbol{f}(t)$ must be white noise with $\left\langle f_{i}(t)\right\rangle=0$ and $\left\langle f_{i}(t) f_{j}(\tau)\right\rangle=2 \Gamma k T \delta_{i j} \delta(t-\tau)$, where $k$ is Boltzman's constant, and $T$ is the temperature in Kelvin, as required by the fluctuation-dissipation theorem. Langevin equations are an adequate description of the kinetics of small classical objects under the influence of Brownian forces. This is the type of description that is required to approximate the fine-scale conformational dynamics of the protein.

Just as discrete Markov models are approximations of the finer scale motions of the protein described by the Langevin equation, the Langevin equation is an approximation of the protein's motion on a still finer scale. The choice of a model therefore depends entirely on the desired range of its validity since the protein has a complex structure on many different scales. The Langevin equations' range of greatest value is where we are interested in the fine-scale motion of the gating mechanism, but not in the very rapid oscillations and movements of the individual atoms in the protein. The generalized coordinate $\boldsymbol{X}$ then represents the gross position of many atoms in the molecule whose average relative position remains fixed, and that move together as a group. For instance, the four S4 channel subunits have been proposed as just such voltage-sensitive moving parts. Each of these four subunits are made up of about 20 amino acids that are believed to take the more or less rigid form of an alpha helix, and contain many regularly spaced charged groups. Gating is believed to involve some repositioning of the S4 subunits in the electric field across the membrane, but how this repositioning works is unknown and as is currently a topic of very intense interest.

Interest is shown in simple models of this repositioning where $\boldsymbol{X}$ is fairly low dimensional: otherwise there is not much point of even trying to model the system at a level intermediate between the 'fast' atomic scale and the 'slow' discrete Markovian scale. The energy $H(X)$ represents the combined intramolecular forces that 
act on a voltage-sensing molecular group, and should be thought as indicative of the structure and function of the protein. Often a single-variable model where $X=\boldsymbol{n} \cdot \boldsymbol{X}$ will be considered, since the greatest changes in energy will occur in the normal direction as a single (perhaps independent) charged group such as the S4 alpha helix moves through the electric field. These very voltage-dependent motions are the ones we can expect to learn the most about with the ultrahigh-frequency NRS method.

Discrete Markov models for gating can be derived from a more detailed Langevin approach when the energy function $H(\boldsymbol{X})$ has a number of wells (labeled by a discrete index) whose depths are large compared with $k T$. That is, the lowest energy state that the channel must pass through to get from state $i$ to state $j$ is at an energy $\Delta U_{i j} \gg k T$ higher than state $i$. In this case the channel will spend the vast majority of the time near the bottom of one of the energy wells, and will only occasionally make a thermally activated transition to another energy well. It will then spend a relatively long time in the new well, before making another transition, and so on. In this case when a system is in a particular energy well it can be said to be in a 'state' corresponding to that energy well. This is not meant to imply that the channel is not constantly in motion, only that if we are interested only in the motion on the scale of the transitions between the energy minima then the protein can be said to be in a particular state that corresponds to the basin of attraction of that energy minima. It can be shown that, when a transition between the wells takes a much longer time than it takes for the system to relax within a given well, then the transitions between the wells are statistically independent, and random. This is all that is required to describe the probability distribution over the three states, and the developments of the distribution in time, provided the transition rates have been specified, in terms of a discrete Markovian process. A complete specification of such a discrete model now requires only a specification of the Eyring transition rates between the states $W_{i j}(t)=W_{i j}(0) \exp \left(q_{i j} V / k T\right)$, where a base rate $W_{i j}(0)$ and a gating charge $q_{i j}$ must be specified for each transition, in contrast to the specification of an entire energy function $H(\boldsymbol{X})$ that is required for the Langevin approach.

These ideas remain valid for time-varying potentials if the potential changes more slowly than the microconformational relaxation. In this case the constant transition rates in the model can be replaced with the time-varying transition rates $W_{i j}(t)=W_{i j}(0) \exp \left(q_{i j} V(t) / k T\right)$. This is the basis of ordinary NRS, as described in detail in [11], as well as in the first part of this paper. On the other hand, if the channel is driven even faster, a response will be produced that depends not only on the discrete conformational properties of the channel gating, but also on the faster, microconformational dynamics. In such cases the full Langevin equation will be required instead of the discrete approximation. This sensitivity to detail implies that information about the details can be assessed from such high-frequency response measurements- the basic idea behind ultrahigh-frequency NRS. It turns out that in such cases the response of the systems can be directly interpreted in terms of a detailed picture of the microscopic dynamics. This surprising result is a consequence of my dynamical reconstruction theorem [17].

\section{The dynamical reconstruction theorem}

The mathematical theories behind optimal control theory (from engineering and applied mathematics) and the theory of large fluctuations (from statistical physics) are nearly identical. The problem begins on the level of the Langevin description of eqn (1), except that, for the time being, the case in which the thermal fluctuations can be neglected will be considered, and $f(t)=0$. Since very large and very rapid pulses of $V(t)$ will only be considered the thermal fluctuations will have little chance to act during the pulse, being relatively small. This is to be contrasted with the long-time influence of the thermal fluctuations: if a long enough time passes a large thermal fluctuation will come along and cause the channel to make a transition to another state. In the present situation by the time such a large fluctuation occurs the experiment (the rapid voltage pulse) is long over. To first order then the influence of the rapid voltage pulse on the channel can be described with the equation $\Gamma \dot{X}=-H^{\prime}(X)+Q E(t)$, where I now consider only the important component of motion $X$ that 
is normal to the electric field. Later it will be shown how small thermal fluctuations during the pulse can be taken into account, and that they will not generally lead to systematic errors.

First consideration will be given to a pure engineering problem, the problem from optimal control theory of finding the way to bring a system from a stable point to some given point requiring the least energy expenditure by the field, where the energy supplied by the driving field is $\Phi=\frac{1}{2} \int_{-\infty}^{0} E^{2}(t) d t$. Here the pulse starts at some point in the past, and is over at $t=0$. This problem also arises in the theory of large fluctuations in statistical physics [19]. The functional $\Phi$ is then proportional to the energy input of the pulse. I want to calculate the 'optimal' pulse that will bring the ion channel to a particular microconformational state $X(0)$ with the least expenditure of energy, that is, that corresponds to the least $\Phi$. The results can be applied much more generally, but for the present purposes I will merely state the answer for this problem: the optimal trajectory $\tilde{X}(t)$ of the channel through the microconformational states is found by integrating the equation of motion $\Gamma \dot{\tilde{X}}=H^{\prime}[\tilde{X}(t)]$ which is in fact the time-reversed equation of motion for the channel in the absence of a driving field, and the optimal pulse itself is given by $\tilde{E}(t)=2 \Gamma H^{\prime}[\tilde{X}(t)] / Q$. The equation of motion for the optimal path is similar to the Langevin equation in the absence of the electric field except that now the potential energy function is inverted. Since I am interested in the situation where the channel starts out near one of the minima of $H(X)$, in the equation for the optimal path the system is at a local maximum. It then goes 'down hill' and this motion $\tilde{X}(t)$ can be used to determine the optimal driving $\tilde{E}(t)$. The optimal path and the optimal driving are unique for each $H(X)$.

I now have everything I need to prove the dynamical reconstruction theorem, which I will state in a very straightforward, and practical way. By making use of the equation for the optimal path and the electric field above, as well as the equation of the dynamics of the system $\Gamma \dot{\tilde{X}}=-H^{\prime}[\tilde{X}(t)]+Q \tilde{E}(t)$, I obtain the following mathematical relations,

$$
H[\tilde{X}(t)]=Q^{2} \int_{-\infty}^{t} \tilde{E}^{2}(\tau) d \tau, \quad \tilde{X}(t)=(Q / 2 \Gamma) \int_{-\infty}^{t} \tilde{E}(\tau) d \tau, \quad \Gamma=(Q / 2 \tilde{X}(0)) \int_{-\infty}^{t} \tilde{E}(\tau) d \tau .
$$

The first two of these can be used to determine the unknown quantity $H(X)$ from the quantities, $Q, \Gamma$, and $\tilde{E}(t)$. The last of these just specifies the coefficient of friction in terms of the final point $\tilde{X}(0)$.

It is possible to rewrite these equations in terms of the quantities that most electrophysiologists currently use. The voltage across the membrane is $V(t)=\lambda E(t)$, where $\lambda$ is the membrane thickness. I introduce the dimensionless coordinate $x=X / \lambda_{i j}$ where $\lambda_{i j}$ is the physical distance between the potential minima of the $i$ th and potential barrier. The gating charge of this transition is given by $q_{i j}=\lambda_{i j} Q / \lambda$. We have $X(0)=\lambda_{i j}$ where the initial point $X(-\infty)$ is set at the origin, and where the energy barriers are $\Delta U_{i j}$. Combining all of these

$$
h[\tilde{x}(t)]=\int_{-\infty}^{t} \tilde{V}^{2}(\tau) d \tau\left[\int_{-\infty}^{0} \tilde{V}^{2}\left(\tau^{\prime}\right) d \tau^{\prime}\right]^{-1}, \quad \tilde{x}(t)=\int_{-\infty}^{t} \tilde{V}(\tau) d \tau\left[\int_{-\infty}^{0} \tilde{V}\left(\tau^{\prime}\right) d \tau^{\prime}\right]^{-1}
$$

is obtained, and where $h(x)=H(x) / \Delta U_{i j}$ is a dimensionless energy function. The gating charge can also be determined uniquely. Since it is known that the $i$-to- $j$ transition has been excited, $q_{i j}=\int_{-\infty}^{0} \tilde{I}_{g}(t) d t$, where $\tilde{I}_{g}(t)$ is the measured gating current during the optimal pulse.

What this section shows is that not only can the standard parameters of interest to electrophysiologists studying channels at the level of a discrete Markov process be unambiguously determined, but details of the microscopic motion, namely the shape of the actual energy function $h(x)$ can be determined from the optimal voltage pulse $\tilde{V}(t)$. While the former amounts to a resolution of the problems of ambiguity that lead to such great difficulties with current electrophysiological techniques, the latter represents a level of information about the channel dynamics that was not even previously contemplated. 


\section{Applying the dynamical reconstruction theorem}

I will now show how the optimal voltage pulses $\tilde{V}(t)$ to excite and control particular transitions in ion channels can be determined experimentally. This can be done even though the microscopic motions of the gating mechanism are not observed directly. It is merely necessary to ascertain that the channel passed through the transition state, that is, to ascertain that the field pulse is the optimal pulse to cause a transition to a new conformational state.

Electrophysiologists already have the ability to observe channels at the level of the conformational states. In the present situation the channels are prepared in the resting state (e.g. $\mathrm{C}_{1}$ in Fig. 1) by applying a large, negative conditioning potential for long enough time. After application of a pulse the percentage of channels in the new state (e.g. $\mathrm{C}_{2}$ in Fig. 1) can be ascertained in the following way. Immediately upon termination of the pulse the voltage is returned to the initial value and the gating current is measured. Channels that were excited to $C_{2}$ will now, over the course of several ms, relax back to state $C_{1}$ via thermal activation over the barrier between them. This will be observed as a pure exponential decay of the gating current for large enough hyperpolarizations of the voltage. The coefficient of this decay is proportional to the probability of inducing a transition via the initial pulse. An experimental search can then be performed for the optimal pulse that leads to a subsequent pure decay of the gating current upon repolarization of the potential.

It still remains to explain how the optimal pulses are determined experimentally. The problem is well defined in the sense that we can merely start guessing the answer, and gradually improve over time since the 'quality' of the guess can be determined experimentally by defining an experimental cost functional $\Psi=\left(k(V(t))-k_{0}\right)^{2}+\int_{-\infty}^{0} V^{2}(t) d t$, where $k(V(t))$ is the coefficient of the exponential decay of the gating current $I_{g}(t)=k \exp (-t / \tau)$ subsequent to application of the pulse $V(t), t \in[-\infty, 0]$, and $k_{0}$ is the maximum amplitude of this decay. This quantity will be zero iff $V(t) \rightarrow \tilde{V}(t)$, so the search might be conducted randomly for the pulse with the smallest $\Psi$. This amounts to a variational principle that can be used to determine the microconformational dynamics of the channel, hence dynamical reconstruction.

However, the search can be performed much more efficiently than that. Guesses $u(x)$ can be generated for the shape of the dimensionless energy function $h(x)$. The optimal pulses for this potential can then be computationally determined, and applied to the ion channel. When the optimal $u(x)$ is found, it is the unknown $h(x)$ sought for. To do this it helps to have a simple way of parametrizing the trial functions $u(x)$. Recalling that $0 \leq x \leq 1$, where $h(0)=0$ and $h(1)=1$, we can use a modified Fourier expansion $u(x)=x+\sum_{n=1}^{\infty} a_{n} \sin (2 \pi n x)$ where the first term controls the rise of the energy function from 0 to 1 , and the rest of the terms control the shape of the function. In practice we can truncate this series at an appropriate point. In this case the potential is described by the $n+1$ parameters $a_{0}, \ldots, a_{n}$, where $n$ can be chosen as large as is needed.

The optimal path is determined by first integrating $\dot{\tilde{x}}=h^{\prime}[\tilde{x}(t)]$ in terms of the dimensionless coordinate and energy, where the dimensionless time $s=t / \tau$ has been introduced where $\tau=\Gamma \lambda_{i j}^{2} / \Delta U_{i j}$. Remember that this equation is the time-reversed version of the regular dynamics (here the sign of the potential energy is reversed). Since the channel starts at an energy minima, it is at a local maxima in the equation for the optimal path. The process of the channel escaping over the energy barrier between the states is then described in this equation as traveling down hill in the time-reversed equation for the optimal path. We then have $\dot{\tilde{x}}=1+\sum_{n=1}^{\infty} 2 \pi n a_{n} \cos (2 \pi n x)$, where $\tilde{x}_{u}$ is the optimal path for the trial potential $u(x)$. The optimal pulse is then given by $\tilde{V}_{u}(t / \tau)=V_{0} h^{\prime}\left[\tilde{x}_{u}(s)\right]=V_{0} \dot{\tilde{x}}_{u}(t / \tau)$, where $V_{0}=2 \Gamma \lambda \Delta U_{i j} / Q \lambda_{i j}$. There are thus two additional parameters, a time $\tau$ and a voltage $V_{0}$ related to the fundamental properties of the channel as described above that go into the search for the optimal pulse $\tilde{V}(t)$. This of course is simply the price that must be paid for the simplification of limiting the search over dimensionless potential and coordinates variables.

Having now guessed a potential in the form of the parameters $\tau, V_{0}, a_{0}, \ldots, a_{n}$ the trial optimal voltage pulse, $\tilde{V}_{u}(t)\left[\tau, V_{0}, a_{0}, \ldots, a_{n}\right]$ is then applied to the channels in a macropatched cell using the electrophysiological apparatus and the corresponding cost function $\Psi\left[\tau, V_{0}, a_{0}, \ldots, a_{n}\right]$ measured. The process is repeated 


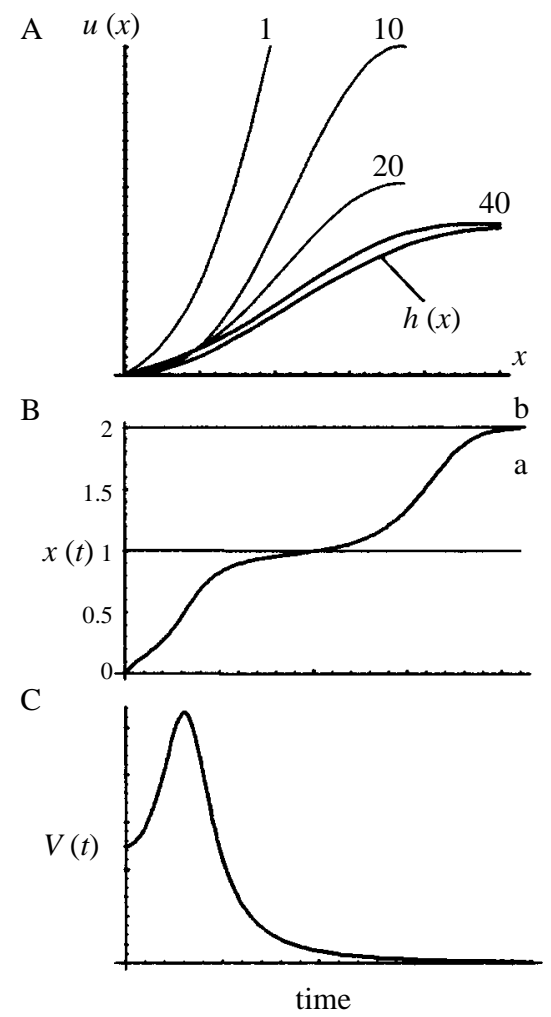

Fig. 4. Numerical illustration of optimal control and dynamical reconstruction showing A, convergence of potential to the correct potential, $\mathrm{B}$, the optimal path and $\mathrm{C}$, the optimal voltage pulse.

many times in a search for the parameters that come as close as possible to $\Psi\left[\tilde{\tau}, \tilde{V}_{0}, \tilde{a}_{0}, \ldots, \tilde{a}_{n}\right]=0$. The $\tilde{\tau}, \tilde{V}_{0}, \tilde{a}_{0}, \ldots, \tilde{a}_{n}$ then determine the quantity we seek, the energy function of the transition from state $i$ to state $j$.

Through an analysis of this problem I have come up with a quite efficient algorithm for determining the optimal parameters. It involves using a simulated annealing genetic search analogous to the one described in [11] (supplementary material). The algorithm makes a random search of parameter space with an everdecreasing search radius. Parameters $\Pi=\left(\tau, V_{0}, a_{0}, \ldots, a_{n}\right)$ are initially chosen at random, or according to some preliminary information, and a number of random variations ('offspring') are generated from this initial set. At the end of each generation the model with the smallest cost $\Psi$ is chosen to act as the new seed for the next generation. Thus there is a survival of the 'fittest', hence the name genetic algorithm. The new parameters $\prod_{k+1}$ at generation $k+1$ are 'bred' from the old parameters $\prod_{k}$ at generation $k$, via the following stochastic rule: $\prod_{k+1}^{i}=\prod_{k}^{i}\left[1+\sigma_{i k} \exp \left(-r_{a} k\right)\right]$ where $\sigma_{i k}$ are uniform random variables on the interval $\sigma_{i k} \in[-\Delta, \Delta]$, where $0 \leq \Delta \leq 1$, and $r_{a}$ is the annealing rate which sets the convergence rate of the algorithm. New values for the random variables are chosen for each parameter indexed by $i$, and for each new generation indexed by $k$. The parameters converge in finite time to nearly fixed values, and the program is terminated after a fixed number of generations.

The technique will be demonstrated by a numerical simulation of the method using the same algorithms. In this case the 'experimental apparatus + ion channels' is model by an equation $\dot{X}=-H^{\prime}(X)+V(t)$. Figure 4A shows a picture of the 'mystery' potential $H(X)$ along with the progressive approximations that 
are made of it using optimal control and dynamical reconstruction. The trial potential was modeled in the form described above with the potential series truncated at $n=2$ terms. Figures 4B and $\mathrm{C}$ show the shapes of the optimal path of the system and the shape of the optimal pulse.

A potential source of error are the small thermal fluctuations in the field that will occur during the application of the pulse. It is easy to see that some of these fluctuations will tend to prevent the channel from making the transition, while others will tend to enhance the transition. Assurance will be needed that these small fluctuations do not bias the transition in such a way that will lead to systematic errors in the determination of the energy function. This problem can be analyzed by considering the effects of small fluctuations about the optimal pulse. I make the substitution $\tilde{E}(t) \rightarrow \tilde{E}(t)+\sqrt{2 \Gamma k T} f(t)$ where $\sqrt{2 \Gamma k T} f(t)$ are the thermal fluctuations required by the fluctuation dissipation theorem as described in the section on Langevin equations. Substituting this into the dynamical equation for the channel gating along with $\tilde{X}(t) \rightarrow \tilde{X}(t)+\epsilon(t)$ where $\epsilon(t)$ is the error caused by the thermal fluctuations the stochastic equation for the error, $\Gamma \dot{\epsilon}=-H^{\prime \prime}[\tilde{X}(t)] \epsilon(t)+$ $Q \sqrt{2 \Gamma k T} f(t)$ is obtained. This is the equation for a thermally fluctuating particle in the totally symmetric, time-dependent potential $\Psi(t, x)=H^{\prime \prime}[\tilde{X}(t)] \epsilon^{2} / 2$. It is easy to see that the average error in such a symmetric potential vanishes, $\langle\epsilon(T)\rangle=0$, so the fluctuations are just as likely to lead to lowering or raising of the required energy. Thus, a more accurate way of defining our cost function would be one where the optimal pulse only leads to half of the population changing states, and where the cost is defined as $\Psi=\left(k-k_{0} / 2\right)^{2}+\int_{-\infty}^{0} V^{2}(t) d t$. The total error should vanish when the new cost function is used when the thermal fluctuations are small since the measurement is made over a large ensemble of channels.

In conclusion it is quite interesting to note, in line with my original goal of describing the 'inverse' of Landauer's blowtorch, that the optimal voltage pulse, and the optimal thermal fluctuation that leads to thermally activated escape, have exactly the same shape. They are in fact equivalent in every way since the calculations that lead to both processes are identical. A thermally activated fluctuation occurs by a random search (by the fluctuating system itself) over all possible fluctuations until the optimal one is found whereas, in the case of optimal control, our search is more confined and more efficient. Thus at the microscopic level it turns out that not only can Landauer's blowtorch principle be inverted, but that nature herself makes use of this fact.

Acknowledgements - I would like to thank Dorothy Hanck who participated in the work described in the first section of this paper, and Richard Benzinger for many helpful suggestions.

\section{References}

[1] R. Landauer, J. Stat. Phys. 53, 233 (1988), and references therein.

[2] R. Landauer, J. Appl. Phys. 33, 2209 (1962).

[3] M. Magnasco, Phys. Rev. Lett. 71, 1477 (1993).

[4] A nice example is the binding of CO in myoglobin, J. O. Alben et al., Phys. Rev. Lett. 44, 1157 (1980); A. Ansari, et al. Proc. Natl. Acad. Sci. USA 82, 5000 (1985); I. E. T. Iben et al. Phys. Rev. Lett. 62, 1916 (1989).

[5] J. D. Watson et al., Recombinant DNA (New York, Scientific American Books, Freeman and Co., 1992).

[6] A. L. Hodgkin and A. F. Huxley, J. Physiol. (Lond.) 116, 424 (1952).

[7] D. Colquhoun and A. G. Hawkes, Proc. Roy. Soc. Lond. B300, 2053 (1981).

[8] F. J. Sigworth, in Single Channel Recording, edited by B. Sakmann and E. Neher, (New York, Plenum, 1995).

[9] G. Marmont, G. J. Cell. Comp. Physiol. 34, 351 (1949); K. S. Cole, Arch. Sci. Physiol. 3, 253 (1949); A. L. Hodgkin, A. F. Huxley, and B. Katz, Arch. Sci. Physiol. 3, 129 (1949); A. L. Hodgkin, A. F. Huxley, and B. Katz, J. Physiol. (Lond.) 116, 424 (1952).

[10] C. M. Armstrong, Physiol. Rev. 61, 644 (1981). 
[11] M. M. Millonas and D. A. Hanck, Biophys. J. 72, A117 (1997); M. M. Millonas and D. A. Hanck, Biophys. J. 74, 210 (1998); Phys. Rev. Lett. 80, 401 (1998).

[12] C. A. Vandenberg and F. Bezanilla, Biophys. J. 60, 1499 (1991); C. A. Vandenberg and F. Bezanilla, Biophys. J. 60, 1511 (1991).

[13] W. Horsthemke and R. Lefever, Noise-induced Transitions: Theory and Application in Physics, Chemistry, and Biology. Springer-Verlag; Biophys. J. 35, 415 (1980).

[14] E. Stefani and F. Bezanilla, Biophys. J. 72, A131 (1997).

[15] J. C. Makielski et al. Biophys. J. 52, 1.

[16] R. S. Maier and D. L. Stein, Phys. Rev. Lett. 71, 1783 (1993); M. I. Dykman, M. M. Millonas, and V. N. Smelianskiy, Phys. Lett. A195, 53 (1994); S. J. B. Einchcomb and A. J. McKane, Phys. Rev. E49, 257 (1994).

[17] M. M. Millonas in Controlling Complex Systems (Sante Fe Institute Press, Addison-Wesley, in press).

[18] E. Stefani and F. Bezanilla, Biophys. J. 72, A131 (1997).

[19] M. M. Millonas, Nature 389, 443 (1997). 